\title{
Preoperative Surgery Planning for Percutaneous Hepatic Microwave Ablation
}

\author{
Weiming Zhai ${ }^{1}$, Jing Xu ${ }^{2}$, Yannan Zhao ${ }^{1}$, Yixu Song ${ }^{1}$, Lin Sheng ${ }^{3}$, and Peifa Jia ${ }^{1}$ \\ ${ }^{1}$ State Key Laboratory of Intelligent Technology and Systems \\ National Laboratory for Information Science and Technology \\ Department of Computer Science and Technology, Tsinghua University, Beijing 100084, China \\ wmzhai02@mails. tsinghua.edu.cn \\ ${ }^{2}$ Department of Precision Instruments and Mechanology \\ Tsinghua University Beijing, 100084, China \\ ${ }^{3}$ Tsinghua University Yuquan Hosiptal, Beijing 100049, China
}

\begin{abstract}
A novel preoperative surgery planning method is proposed for percutaneous hepatic microwave ablation. An iterative framework for necrosis field simulation and 3D necrosis zone reconstruction is introduced here, and the necrosis model is further superimposed to patient anatomy structures using advanced GPU-accelerated visualization techniques. The full surgery planning is performed by the surgeon in an interactively way, until the optimal surgery plan is achieved. Experiments have been performed on realistic patient with hepatic cancer and the actual necrosis zone are measured in postoperative CT images for patient. Results show that this method is relative accurate for preoperative trajectory plan and could be used as an assistant to the clinical practice.
\end{abstract}

Keywords: surgery planning, minimal invasive surgery, hepatic microwave ablation, necrosis field.

\section{Introduction}

Hepatic cancer is one of the most common malignant neoplasms and is responsible for more than one million deaths per year worldwide [1]. Partial resection remains the main option for treatment of hepatic cancer; however, it's only suitable for 9-27\% of patients [2], primarily due to tumor location or underlying liver disease. Therefore, minimal invasive therapy is essential to improve the prognosis in hepatic cancer patients.

Microwave ablation is an effective minimal invasive therapy for hepatic cancer, which exhibits many advantages over other alternatives to resection [3]. It shares the common advantages of thermal ablation techniques such as flexible treatment approach, good tolerability and the ability to consistently create reproducible and predictable necrosis areas. However, the surgery planning method for microwave ablation is seldom discussed compared to the hot topic of surgery planning in radio-frequency ablation as shown

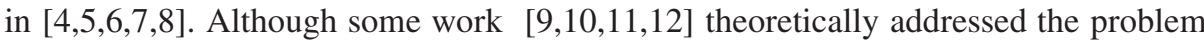
of modeling the thermal field of microwave ablation, a surgery planning method for percutaneous microwave ablation is absent in clinical practice.

D. Metaxas et al. (Eds.): MICCAI 2008, Part II, LNCS 5242, pp. 569-577, 2008.

(C) Springer-Verlag Berlin Heidelberg 2008 
In this paper, we proposed a novel framework for the preoperative planning of percutaneous hepatic microwave ablation surgery. The necrosis field of the ablation is computed with an adaptable method based on surgery trajectories, and then the 3D model of the necrosis zone is reconstructed and superimposed to the patient anatomy structures using advanced visualization techniques. And the clinical surgeon could interactively adjust the trajectories and predict the necrosis zone, until the surgery is satisfied.

The rest of this paper is organized as following. Section 2 describes the system architecture and the key methods used in this system. Section 3 gives out the simulation results on clinical surgeries. Finally, section 4 is devoted to the discussion of conclusion and future works.

\section{Methods}

The flowchart of the system is shown in Fig. 1] Firstly, the preoperative patient images are used to generate 3D patient anatomy structures, and then surgery trajectories are superimposed to the 3D scene. Afterward, the necrosis field cause by microwave energy is predicted and the necrosis zone is reconstructed to 3D mesh model, which is further visualized in $3 \mathrm{D}$ scene. The computation and visualization process is implemented with GPU-accelerated algorithms, which could minimize the computation time, so that the surgeon could iteratively adjust the trajectories and see the predicted result, until the optimal surgery plan is achieved. Note that during microwave ablation, the thermal properties and the blood perfusion rate change with temperature elevation and thermal necrosis, and the temperature field is then influenced by the changed properties. So in order to better satisfy the realistic condition, we couple the calculation of the temperature field, the necrosis field and the dynamic changing properties in an iterative schema, so as to give more accurate results.

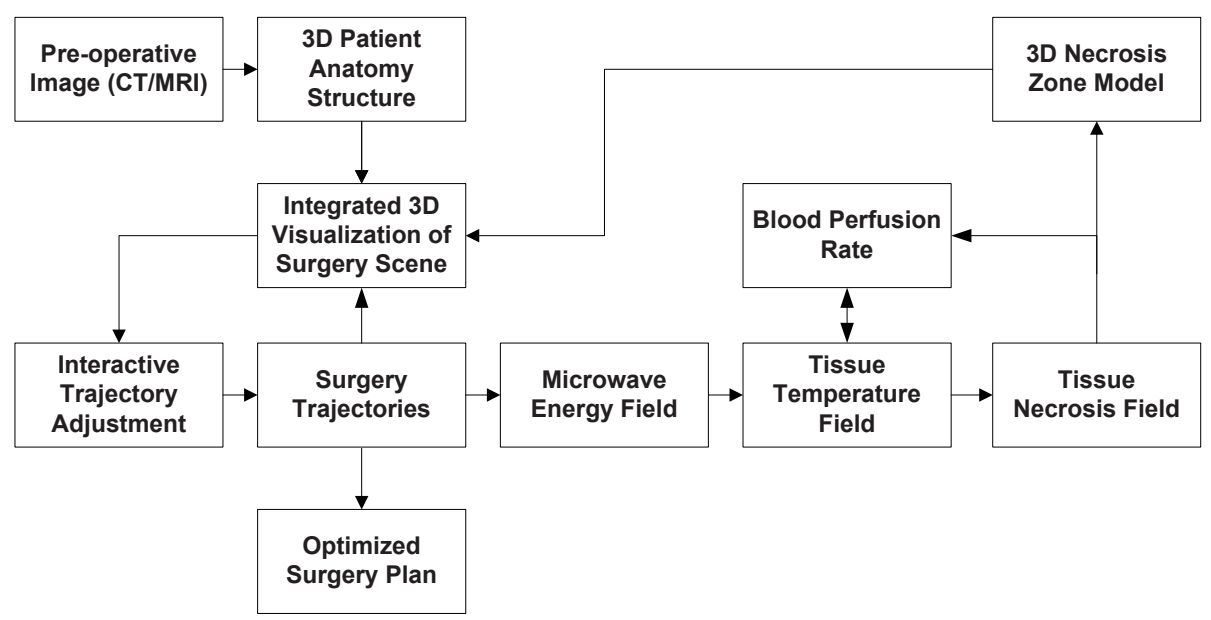

Fig. 1. Flowchart of the Surgery Planning System 


\subsection{Calculation of Microwave Energy Field}

To calculate the specific absorption rate (SAR) of the microwave energy, we used the equation proposed by [11]

$$
S A R=C_{t} W \frac{\left[2 \varepsilon r+(N-2) e^{-2 \varepsilon r}\right]}{r^{2}} e^{-z^{2} / z_{0}^{2}}
$$

Where $W$ is the microwave power and $C_{t}$ is a scale constant, and this assumption is based on that $S A R$ is proportional to the microwave power; $N$ is an exponential constant, and it should be 2.2 here; $r$ is the radial distance and $z$ is the axial distance. This equation is a semi-empirical formula and the parameter $C_{t}$ and $Z_{0}$ may be change according to the tissue type and different thermotherapy conditions while keeping the equation's framework unchanged, and an optimization algorithms is needed to revised these parameters according to simulation results from clinical practice.

\subsection{Calculation of Tissue Temperature Field}

Calculation of the tissue temperature field requires the evaluation of temporal and spatial distributions of temperature. This class of problems has been traditionally addressed using the Pennes bioheat transfer equation [13 14,15], which accounts for the ability of tissue to remove heat by both diffusion and perfusion of blood

$$
\rho c \frac{\partial(T)}{\partial t}=\frac{\partial}{\partial x}\left(\lambda \frac{\partial T}{\partial x}\right)+\frac{\partial}{\partial y}\left(\lambda \frac{\partial T}{\partial y}\right)+\frac{\partial}{\partial z}\left(\lambda \frac{\partial T}{\partial z}\right)+\rho_{b} \rho_{c} w_{b}\left(T_{b}-T\right)+Q_{r}+Q_{m}-Q_{c}
$$

Where $T$ is the local thermodynamic temperature of biological tissue; $\rho$ is the density of biological tissue; $c$ is the specific heat of biological tissue; $\lambda$ is the thermal conductivity of biological tissue; $\rho_{b}$ is the density of arterial blood; $c_{b}$ is the specific heat of arterial blood; $T_{b}$ is the thermodynamic temperature of arterial blood; is the blood perfusion rate; $Q_{r}$ is the microwave energy absorbed by biological tissue in unit time of unit volume, which is the $S A R$ in (1); $Q_{m}$ is the metabolic heat and $Q_{c}$ is the conductive heat between microwave antenna and biological tissue. The value of $Q_{m}$ and $Q_{c}$ is comparatively small and ignored.

According to [16], the thermal properties of the biological tissue changes with the temperature, and it can be approximated according to the water content and the dynamic changes in the thermal properties of the water

$$
\left\{\begin{array}{l}
c(t)=4190\left(0.37+0.63 k_{c} w\right) \\
\lambda(t)=0.419\left(0.133+1.36 k_{\lambda} w\right)
\end{array}\right.
$$

Where $w$ is the water content of tissue, which is about 0.69 for liver; $t$ is the temperature of tissue; $k_{c}$ and $k_{\lambda}$ are the specific heat and the thermal conductivity of water with respect to the change of temperature respectively (the temperature may scale from $20^{\circ} \mathrm{C}$ ) to $\left.100^{\circ} \mathrm{C}\right)$ ). And their value may be derived from

$$
\left\{\begin{array}{l}
k_{c}=1+1.016 \times 10^{-4}(t-20) \\
k_{\lambda}=1+1.78 \times 10^{-3}(t-20)
\end{array}\right.
$$


During the ablation process, the blood perfusion rate of biological tissue first become higher with the temperature elevation as a result of vascular expansion and blood flow acceleration, and then it will become lower when the blood is thermally coagulated. According to [17], similar result for dynamic changes in blood perfusion rate $\omega_{b}$ with respect to temperature and the degree of necrosis can be formulated as:

$$
\omega(T, \Omega)=\omega_{b 0} f_{t} f_{u}
$$

Where $\omega_{b 0}$ is the initial perfusion rate of the tissue, which is about $0.0182 \mathrm{~m}^{3} \mathrm{~s}^{-1} \mathrm{~m}^{-3}$ for liver; $f_{t}$ is the scale coefficient of perfusion rate with the change of temperature

$$
f_{t}= \begin{cases}4+0.6(t-42) & 37 \leq t \leq 42, \Omega<1 \\ 4 & t \geq 42, \Omega<1 \\ 0 & \Omega \geq 1\end{cases}
$$

The undamaged tissue fraction $f_{u}$ is expressed as

$$
f_{u}=\exp (-\Omega)
$$

Where $\Omega$ is the necrosis function that indicates the thermal necrosis field in the hepatic tissue, and it can be calculated by the Arrhenius equation.

\subsection{Prediction of Tissue Necrosis Zone}

The key step for the surgery plan is to predict the necrosis zone, where the cancerous cells are thermally damaged by the microwave energy. Currently, most of the clinical surgeons only use the temperature of biological tissue (generally it should be $60^{\circ} \mathrm{C}$ ) to judge whether the tissue is thermally damaged. However, this type of method is not accurate in most circumstances, because the thermal damage of biological tissue is not only related to the tissue temperature, but also be related to the lasting time for the tissue exposed to that temperature. In order to precisely predict the necrosis area, especially when we encounter the problems that 'how long the tissue will be thermally damaged under $54^{\circ} \mathrm{C}$ ?' or 'below which temperature the tissue will never be thermally damaged?', the Arrhenius equation [1819] is used to estimate cumulative thermal damage and the necrosis field

$$
\Omega=\int_{0}^{\tau} A \exp \left(-\frac{\Delta E}{R T}\right) \mathrm{d} \tau
$$

Where $A$ is the exponential factor; $\Delta E$ is the activation energy; $R$ is the universal gas constant, and the default value for them are $9.4 \times 10^{104} \mathrm{~s}^{-1}, 6.68 \times 10^{5} \mathrm{~J} \cdot \mathrm{mol}^{-1}$ and $8.31 \mathrm{~J} \cdot \mathrm{mol}^{-1} \cdot \mathrm{K}^{-1}$ respectively. The necrosis is irreversible and the tissue cell is thermally damaged if $\Omega$ is more than 1 .

Note that during microwave ablation, the thermal properties as well as the blood perfusion rate change with temperature elevation and thermal damage, with the energy and temperature fields then influenced by the changed properties. So in order to better satisfy the realistic condition, we couple the calculations of the temperature field, the necrosis field and the dynamic changing properties in an iterative method, so as to give more accurate results. 


\subsection{Adjustment of Trajectory Plan}

The surgery planning process consists of choosing the best strategy among a lot of candidate trajectories, taking into account the whole anatomy of the patient. For each considered trajectory, the corresponding necrosis field is predicted, and then the $3 \mathrm{D}$ model of the necrosis zone is reconstructed and superimposed onto the patient's anatomy as shown in Fig. 2. The 3D mesh model of the necrosis zone is reconstructed using the GPU-accelerated marching tetrahedron (MT) algorithm [20] by extracting the $\Omega=1$ isosurface in necrosis field. Meanwhile, the patient anatomy structure is rendered by GPU raycasting volume rendering algorithms [21] using the data from CT images. Both of these algorithms are implemented in HLSL using DirectX10 shaders, which may dramatically reduce the compuatation time.

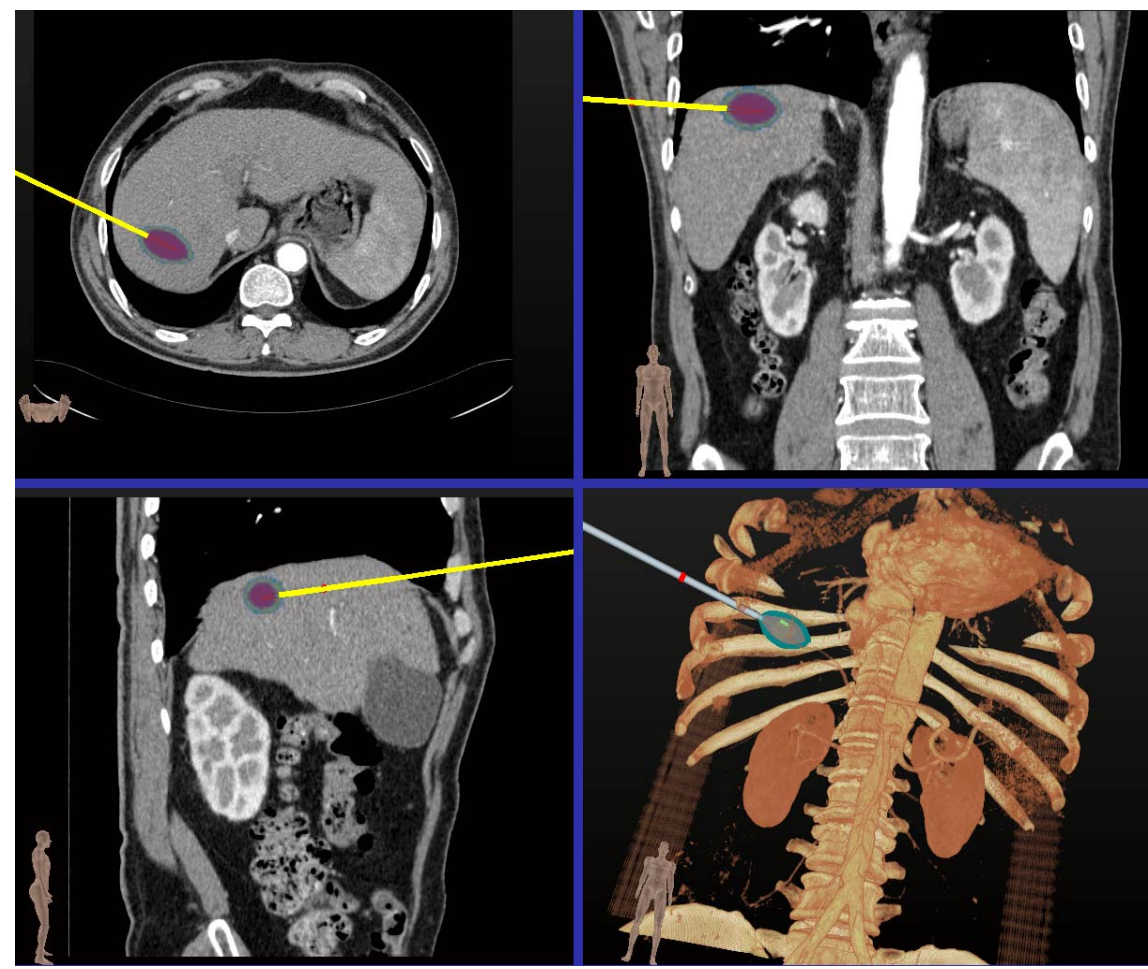

Fig. 2. Interactive Surgery Planning

The trajectory planning is performed in an iterative manner; the surgeons adjust the trajectories until the optimized condition is satisfied. To adequately destroy the entire tumor, the necrosis field should extend $5-10 \mathrm{~mm}$ beyond the tumor boundary [22]. And to envelope the entire tumor with a safety margin, multiple microwave antenna trajectories with different pose, power and duration time could be applied concurrently or 
continuously to the same patient, which could leads to larger necrosis zones [2324]. The surgeons also need to make sure the selected trajectories do not intersection with bone and large vessels, and the necrosis zone should not cover any risk tissue of the patient that could threat the patient's life.

\section{Experiments and Results}

The method has been evaluated on realistic percutaneous microwave ablation surgeries for hepatic cancer. For all these surgeries, a computer with Intel CoreDuo 2.4G CPU, 2G RAM and Nvidia GeForce 8800GTS Graphics Card is used to simulate the necrosis field and render the 3D scene as shown in Fig. 2, the typical calculation time for the necrosis field took less than 2 second, and the rendering time typical reaches to $20 \mathrm{fps}$ which could provide a real-time interactive user interface for surgeons in trajectory planning. The UMC-I microwave ablation equipment from Institute 207 of Aerospace Industry Company is used in the surgeries, and the equipment is operated at $2,450 \mathrm{MHz}$.

For all these patients, preoperative abdominal CT is acquired before surgeries. Then, the surgery trajectories are adjusted by the surgeon and the necrosis zone is reconstructed and rendered with the anatomy structures of the patient. During the operate procedure, the trajectory angle, the puncture point on the patient skin and the puncture depth of the microwave antenna are used to guide the surgeon for the right pose. And the power and duration time for each trajectory are also used as a advice for the operation. Noting that the simulation may not fully identical to the realistic situation and the surgeon may not precisely put the needle to the exactly right place, iron-constantan thermocouples are used to measure the temperature of the tissue, and realtime ultrasound image is used to guide the placement of all these instrument. About 5 days to 2 weeks later, the postoperative abdominal CT is scanned for the patient, and the realistic necrosis zone is measured CT and compared to the simulated data. The results for postoperative evaluation are listed in Table 1 the measured sizes of necrosis zones in postoperative CT and the simulated sizes in surgery planning are listed and compared in

Table 1. Results for Postoperative Evaluation

\begin{tabular}{|c|c|c|c|c|}
\hline No. & Measured Size $\left(\mathrm{cm}^{3}\right)$ & Simulated Size $\left(\mathrm{cm}^{3}\right)$ & Simulation Error $(\%)$ & Measuring Date \\
\hline 1 & 4.4 & 4.7 & +6.8 & 5 \\
\hline 2 & 5.9 & 6.0 & +1.7 & 7 \\
\hline 3 & 11.1 & 12.2 & +9.9 & 6 \\
\hline 4 & 14.9 & 14.8 & -0.7 & 7 \\
\hline 5 & 17.9 & 16.1 & -10.0 & 7 \\
\hline 6 & 18.0 & 16.7 & -7.2 & 7 \\
\hline 7 & 22.5 & 23.5 & +4.4 & 12 \\
\hline 8 & 24.6 & 22.3 & -9.3 & 10 \\
\hline 9 & 23.8 & 20.7 & -13.0 & 9 \\
\hline
\end{tabular}




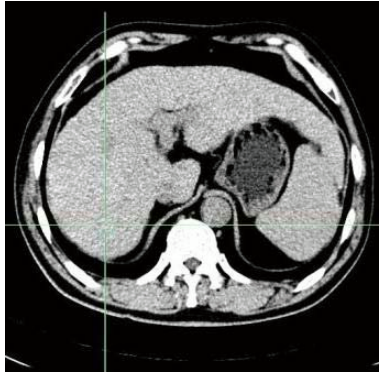

(a) Preoperative $\mathrm{CT}$

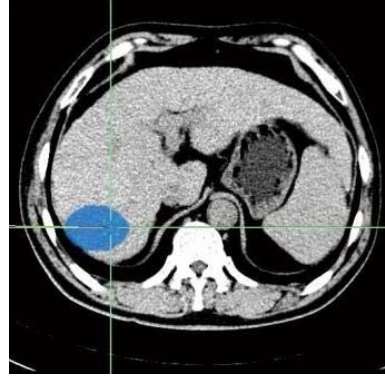

(b) Surgery Plan in 2D

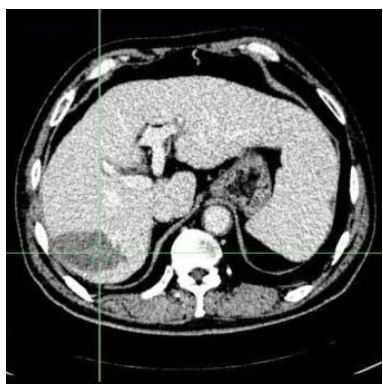

(c) Postoperative CT

Fig. 3. Clinical Result on Patient with Hepatic Cancer

the table. The measured data in clinical practice is used to further modify the parameters $C_{t}$ and $z_{0}$ used in equation (1) for better accuracy.

Fig. 3 shows a group of CT image of of the same patient for a typical surgery. Fig 3(a) is the preoperative CT of the patient, the tumor located at right lobe of the liver and the rib blocks this area for normal trajectories. A carefully designed surgery plan is taken and the simulation on the axial slice is shown in Fig 3(b) The surgery is performed on the patient in less than 10min, and the postoperative CT in Fig 3(c) shows that the entire tumor is destroyed and no risky tissue is hurt.

\section{Conclusion and Future Work}

In this paper, we proposed a novel surgery planning method for percutaneous hepatic microwave ablation. The surgery planning process is performed in an interactive manner until the surgeon is satisfied with the surgery plan. Experiment results show that the method may simulate in most cases and the planned trajectories could be used as a guidance for the realistic procedure, however, additional assistance instrument such as realtime ultrasound and temperature measurement instrument are also needed in the surgery.

In the future, the surgery planning method could be extended to cancers other than hepatic, such as the lung cancer and breast cancer, and this work could be done in the nearly future by adaptively revise the parameter in the framework by extensive experiment on the specific tissue. Semi-automatic trajectory optimization method could also be integrated to this framework to automatically provide the surgeon with several candidate trajectories, which could improve the effect of clinical practice.

\section{References}

1. Esquivel, C., Keeffe, E., Garcia, G., Imperial, J., Millan, M., Monge, H., So, S.: Hepatic neoplasms: advances in treatment. J. Gastroenterol Hepatol. 14, 27-41 (1982)

2. Lai, E., Fan, S., Lo, C., Chu, K., Liu, C., Wong, J.: Hepatic resection for hepatocellular carcinoma: an audit of 343 patients. Ann. Surg. 221, 291-298 (1995) 
3. Liang, P., Wang, Y.: Microwave Ablation of Hepatocellular Carcinoma. Oncology 72, 124 131 (2007)

4. Altrogge, I., Kroger, T., Preusser, T., Bskens, C., Pereira, P.L., Schmidt, D., Weihusen, A., Peitgen, H.-O.: Towards Optimization of Probe Placement for Radio-Frequency Ablation MICCAI. In: Larsen, R., Nielsen, M., Sporring, J. (eds.) MICCAI 2006. LNCS, vol. 4190, pp. 486-493. Springer, Heidelberg (2006)

5. Baegert, C., Villard, C., Schreck, P., Soler, L.: Multi-criteria Trajectory Planning for Hepatic Radiofrequency Ablation. In: Ayache, N., Ourselin, S., Maeder, A. (eds.) MICCAI 2007, Part II. LNCS, vol. 4792, pp. 676-684. Springer, Heidelberg (2007)

6. Villard, C., Baegert, C., Schreck, P., Soler, L., Gangi, A.: Optimal Trajectories Computation Within Regions of Interest for Hepatic RFA Planning MICCAI. In: Duncan, J.S., Gerig, G. (eds.) MICCAI 2005. LNCS, vol. 3750, pp. 49-56. Springer, Heidelberg (2005)

7. Villard, C., Soler, L., Gangi, A.: Radiofrequency ablation of hepatic tumors: simulation, planning, and contribution of virtual reality and haptics Computer Methods in Biomechanics and Biomedical Engineering, vol. 8, pp. 215-227 (2005)

8. Villard, C., Soler, L., Gangi, A., Mutter, D., Marescaux, J.: Towards realistic radiofrequency ablation of hepatic tumors 3D simulation and planning. In: Proceedings of SPIE, vol. 5367, pp. 586-595 (2004)

9. Garnier, C., Lafon, C., Dillenseger, J.L.: 3-D Modeling of the Thermal Coagulation Necrosis Induced by an Interstitial Ultrasonic Transducer. IEEE Trans. Bio-Med. Eng. 55, 833-837 (2008)

10. Xu, R., Zhang, Y., Ma, M., Xia, J., Liu, J., Guo, Q., Gu, N.: Measurement of Specific Absorption Rate and Thermal Simulation for Arterial Embolization Hyperthermia in the MaghemiteGelled Model. IEEE Trans. Magnetics 43, 1078-1085 (2007)

11. Zhu, L., Xu, L.X., Chencinski, N.: Quantification of the 3-D Electromagnetic Power Absorption Rate in Tissue During Transurethral Prostatic Microwave Thermotherapy Using Heat Transfer Model. IEEE Trans. Bio-Med. Eng. 45, 1163-1172 (1998)

12. Liu, J., Zhu, L., Xu, L.X.: Studies on the Three-Dimensional Temperature Transients in the Canine Prostate During Transurethral Microwave Thermal Therapy. Transactions of the ASME 122, 372-379 (2000)

13. Pennes, H.H.: Analysis of tissue and arterial blood temperatures in the resting human forearm. J. Appl. Physiol. 1, 93-122 (1948)

14. Wissler, E.H.: Pennes’ 1948 paper revisited. J. Appl. Physiol. 85, 35-41 (1998)

15. Lang, J., Erdmann, B., Seebass, M.: Impact of nonlinear heat transfer on temperature control in regional hyperthermia. IEEE Trans. Bio-Med. Eng. 46, 1129-1138 (1999)

16. Muller, G., Roggan, A.: Laser-induced interstitial thermotherapy, pp. 83-189. SPIE Optical Engineering Press, Bellingham (1995)

17. Jiang, S.C., Zhang, X.X.: Effects of dynamic changes of tissue properties during laser-induced interstitial thermotherapy (LITT). Lasers in Medical Science 19, 197-202 (2005)

18. Diller, K.R.: The mechanisms and kinetics of heat injury accumulation. In: Lee, R.C., Capelli-Schellpfeffer, M., Kelly, K.M. (eds.) Electrical Injury: A Multidisciplinary Approach to Therapy, Prevention, vol. 720, pp. 38-55. NY Acad. Sci., New York (1994)

19. Bhowmick, S., Swanlund, D.J., Bischof, J.C.: Supraphysiological thermal injury in Dunning AT-1 prostate tumor cells. J. Biomech. Eng. 122, 51-59 (2000)

20. Treece, G.M., Prager, R.W., Gee, A.H.: Regularised marching tetrahedra: improved isosurface extraction Computers and Graphics, vol. 23, pp. 583-598 (1999)

21. Scharsach, H.: Advanced GPU Raycasting. In: Proceedings of CESCG 2005 (2005) 
22. Goldberg, S., Gazelle, G., Mueller, P.: Thermal ablation therapy for focal malignancy: a unified approach to underlying principles, techniques, and diagnostic imaging guidance. AJR 174, 323-330 (2000)

23. Kuang, M., Lu, M., Xie, X., Xu, H., Mo, L., Liu, G., Xu, Z., Zheng, Y., Liang, J.: Liver cancer: increased microwave delivery to ablation zone with cooled-shaft antenna: experimental and clinical studies. Radiology 242, 914-924 (2007)

24. Sato, M., Watanabe, Y., Kashu, Y., Nakata, T., Hamada, Y., Kawachi, K.: Sequential percutaneous microwave coagulation therapy for liver tumors. Am. J. Surg. 175, 322-324 (1998) 